\title{
ESCOLITÍNEOS (CURCULIONIDAE: SCOLYTINAE) ASSOCIADOS A PLANTIO DE Eucalyptus urophylla $x$ Eucalyptus grandis NA AMAZÔNIA MERIDIONAL EM ALTA FLORESTA, MATO GROSSO
}

\author{
BARK BEETLE (CURCULIONIDAE: SCOLYTINAE) IN Eucalyptus urophylla $x$ Eucalyptus grandis \\ PLANTATION IN SOUTHERN AMAZON IN ALTA FLORESTA, MATO GROSSO STATE
}

\author{
Marcelo Monteiro ${ }^{1}$ Camila Craus Carvalho ${ }^{2}$ Juliana Garlet $^{3}$
}

\begin{abstract}
RESUMO
Os escolitíneos contribuem no processo de decomposição de árvores caidas ou recém-abatidas e podem ser potencialmente prejudiciais em ambientes florestais, sejam estes naturais ou plantados. Assim, este trabalho teve por objetivo avaliar uma assembleia de Escolitíneos (Curculionidae: Scolytinae) em um plantio de Eucalyptus urophylla x Eucalyptus grandis na Amazônia Meridional, no município de Alta Floresta, no período de agosto de 2015 a agosto de 2016. Foram utilizadas 12 armadilhas de impacto iscadas com etanol, sendo realizadas coletas quinzenalmente. Os insetos coletados foram encaminhados ao Laboratório da UNEMAT, para realização da triagem, sendo enviados para a Universidade Federal do Paraná, para correta identificação das espécies amostradas. Foram coletados 1.248 indivíduos, com um total de 13 espécies em seis gêneros, com maior representatividade: Cryptocarenus, Hypothenemus e Xyleborus. Na análise faunística Criptocarenus diadematus Eggers, Criptocarenus heveae (Hagedorni) e Criptocarenus seriatus Eggers ocorreram como dominantes, muito abundantes, muito frequentes e constantes, com distribuição agregada, podendo ser considerados potenciais insetos-praga em florestas plantadas na região.
\end{abstract}

Palavras-chave: armadilha etanólica; entomologia florestal; coleobrocas.

\begin{abstract}
Bark beetle contributes to the decomposition process of fallen or newly felled trees and are potentially harmful in forest environments, whether natural or planted. The objective of this work was to evaluate an assembly of bark beetle (Curculionidae: Scolytinae) in a planting of Eucalyptus urophylla $x$ Eucalyptus grandis in Southern Amazonia, in the municipality of Alta Floresta, from August 2015 to August 2016. Twelve ethanol-trapped impact traps were used, and collections were performed biweekly. The collected insects were sent to the UNEMAT Laboratory, where they were sorted and sent to the Federal University of Paraná, for the correct identification of the species sampled. A total of 1,248 individuals were collected, with a total of 13 species in six genera, most representative of Cryptocarenus, Hypothenemus and Xyleborus. In the faunistic analysis Criptocarenus diadematus Eggers, Criptocarenus heveae (Hagedorni) and Criptocarenus seriatus Eggers occurred as dominant, very abundant, very frequent and constant, with aggregate distribution, being considered as potential insect pests in forests planted in the region.
\end{abstract}

Keywords: ethanolic trap; forest entomology; bark beetles.

1 Biólogo, Engenheiro Florestal, Mestre em Biodiversidade e Agroecossistemas Amazônicos, Universidade do Estado de Mato Grosso, Av. Perimetral Rogério Silva, s/n, Jardim Flamboyant, CEP 78580-000, Alta Floresta (MT), Brasil. marcelobioengflorestal@gmail.com

2 Engenharia Florestal, Universidade do Estado do Mato Grosso, Av. Perimetral Rogério Silva, s/n, Jardim Flamboyant, CEP 78580-000, Alta Floresta (MT), Brasil. camilacrausef@gmail.com

3 Engenheira Florestal, Dr ${ }^{\mathrm{a}}$, , Professora Adjunta da Faculdade Ciências Biológicas e Agrarias, Universidade do Estado de Mato Grosso, Campus II, Av. Perimetral Rogério Silva, s/n, Jardim Flamboyant, CEP 78580-000, Alta Floresta (MT), Brasil. julianagarlet@unemat.br

Recebido para publicação em 15/03/2017 e aceito em 16/05/2017

Ci. Fl., v. 28, n. 3, jul. - set., 2018 


\section{INTRODUÇÃO}

O Brasil possui uma área de 7,8 milhões de hectares de florestas plantadas, sendo que os plantios de Eucalyptus respondem por 5,6 milhões de hectares, o que representa 72\% do total, e estão localizados, principalmente, nos estados de Minas Gerais, São Paulo e Mato Grosso do Sul. Nos últimos cinco anos, o crescimento da área de eucalipto foi de 2,8\% ao ano, ocupando lugar de destaque, o segmento de celulose e papel (INDÚSTRIA BRASILEIRA DE ÁRVORES, 2016). Além da grande importância econômica e social, os plantios florestais contribuem para a preservação ambiental e para a conservação de espécies arbóreas e de ecossistemas, pois minimizam a pressão extrativista sobre florestas nativas (FONSECA et al., 2010), formando verdadeiros corredores ecológicos para a fauna regional.

A importância econômica do eucalipto decorre do seu crescimento acelerado, capacidade produtiva, adaptabilidade á diversos ambientes e, principalmente, da diversidade de espécies e híbridos, o que torna possível atender à demanda de grande parte dos segmentos que utilizam produtos de origem florestal, principalmente, para as indústrias de papel e celulose (BACHA; BARROS, 2004; GARLET et al., 2009).

Contudo, com o crescimento de áreas com plantios florestais é necessário realizar o monitoramento constante da entomofauna associada a estes, visto que há um grande número de espécies-praga registradas, e a Classe Hexapoda apresenta vários grupos considerados bioindicadores, pois possuem grande diversidade de espécies, habitat e apresentam significativa importância nos processos biológicos dos ecossistemas (BERTI FILHO, 1995). Os insetos são organismos sensíveis a alterações nos ambientes florestais e são considerados bons indicadores ambientais, caracterizando inclusive a qualidade de cobertura do solo (MALUCHE et al., 2003).

Dessa forma é de suma importância o monitoramento de insetos em plantios florestais cultivados, pois é onde estão os maiores problemas entomológicos. Além disso, ressalta-se a importância de conhecer a entomofauna geral destas áreas, buscando identificar predadores, parasitoides e, até mesmo, avaliar a qualidade ambiental destes locais por meio dos insetos bioindicadores, principalmente na Amazônia Meridional que carece de informações sobre insetos associados a ambientes florestais, sejam nativos ou cultivados.

Entre os grupos que merecem destaque em monitoramentos da entomofauna estão os escolitíneos (Curculionidae: Scolytinae), que são pequenos besouros broqueadores, conhecidos como "besourosde-casca" e "besouros-da-ambrosia" e que vivem nas cascas de árvores, logo na superfície da madeira alimentando-se do suculento tecido do floema (WOOD, 1982). Embora muitas das espécies xilófagas ataquem somente árvores doentes, várias espécies são consideradas importantes pragas de cultivos florestais. Além dos danos que causam diretamente às plantas, os escolitíneos são importantes vetores de viroses, além de algumas espécies apresentarem associações com fungos fitotóxicos (LIMA, 1956).

Para o monitoramento dos Scolytinae, no Brasil, a maioria dos pesquisadores utiliza armadilhas iscadas com etanol nos levantamentos populacionais, devido à madeira em processo de fermentação produzir uma série de compostos químicos semelhantes (NAKANO; LEITE, 2000).

Estudos sobre coleobrocas presentes em ambientes florestais com armadilhas na Amazônia Meridional, e no estado de Mato Grosso são poucos, podendo citar Dorval (2002) que realizou levantamento com escolitíneos coletados em Eucalyptus spp. e Silva (2009) que avaliou a entomofauna em diferentes ambientes florestais no norte do Estado com o intuito de analisar os impactos das alterações ambientais na biodiversidade e na quantidade dos insetos (MEURER et al., 2013).

Desta forma, objetivou-se neste trabalho avaliar uma assembleia de Escolitíneos (Curculionidae: Scolytinae) em um plantio de Eucalyptus urophylla x Eucalyptus grandis na Amazônia Meridional em Alta Floresta, Mato Grosso.

\section{MATERIAIS E MÉTODOS}

O estudo foi realizado no período de agosto de 2015 a agosto de 2016, em um plantio de Eucalyptus urophylla x Eucalyptus grandis localizado na Amazônia Meridional na região norte do estado do Mato Grosso, distante $880 \mathrm{~km}$ de Cuiabá, no município de Alta Floresta-MT. 
Conforme Alvares et al. (2014), o clima da região é do tipo Aw com estação seca de inverno. A temperatura média anual fica acima de $26^{\circ} \mathrm{C}$ com precipitação anual entre 2.800 a $3.100 \mathrm{~mm}$.

$\mathrm{O}$ monitoramento foi realizado em um plantio de Eucalipto que corresponde a dois talhões com cinco hectares, pertencentes ao híbrido Eucalyptus urophylla X Eucalyptus grandis com sete anos de idade, implantados com espaçamento de 3 x 2 (três metros entre linhas e dois entre plantas), por meio de armadilhas etanólicas de impacto, modelo Carvalho 47 (CARVALHO, 1998) com algumas adaptações (GONÇALVES et al., 2014). Utilizou-se o álcool 92,8\% GL como isca atrativa. Na porção inferior da armadilha foi acoplado um recipiente plástico, para armazenamento dos insetos coletados, totalizando 12 armadilhas, sendo a distância entre armadilhas de 50 metros, estando a 100 metros entre transectos, conforme a Figura 1. Estas foram instaladas a 1,5 $\mathrm{m}$ do solo, sendo a altura de coleta recomendada para escolitíneos (DORVAL; PERES FILHO; MARQUES, 2004). Quinzenalmente, os insetos eram retirados e a isca e a solução preservante renovadas.

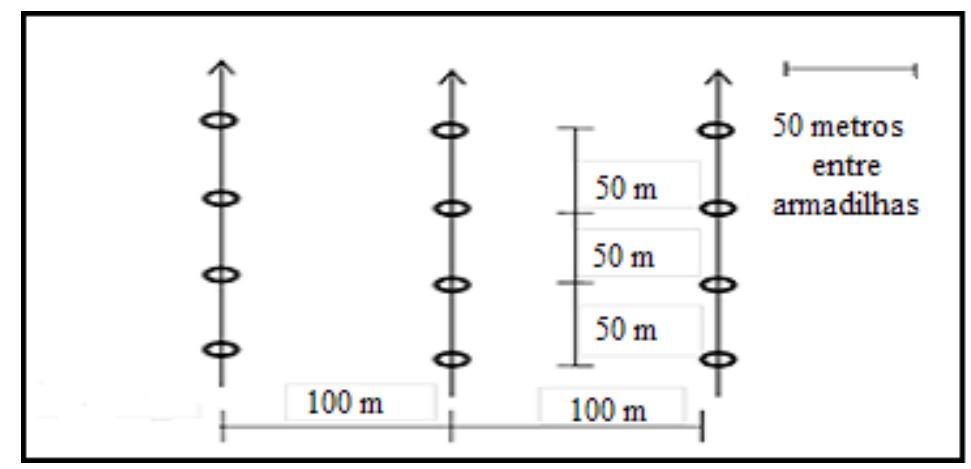

FIGURA 1: Croqui da distribuição das armadilhas na plantação de Eucalyptus urophylla x Eucalyptus grandis, Alta Floresta-MT, 2015/2016.

FIGURE 1: Sketch of the distribution of traps in Eucalyptus urophylla $x$ Eucalyptus grandis planting, Alta Floresta, MT state, Brazil, 2015/2016.

Os indivíduos foram quantificados por contagem direta dos exemplares. As anotações das quantidades de insetos foram efetuadas em ficha apropriada, contendo os dados do trabalho, tais como: local de coleta, data da coleta e localização das armadilhas. Os insetos coletados foram acondicionados em recipientes plásticos devidamente identificados e encaminhados ao Laboratório da Universidade do Estado de Mato Grosso, Campus Alta Floresta, onde se realizou a triagem e separação do material. Posteriormente, foram encaminhados à Universidade Federal do Paraná, para identificação das espécies amostradas pelo Professor Doutor Eli Nunes Marques.

Foram utilizados dados diários meteorológicos de temperatura e umidade relativa sendo transformados em média quinzenal, e realizando-se assim também com os dados da precipitação, para avaliar a influência das variáveis ambientais sobre a assembleia de escolitíneos. As informações foram obtidas da Estação Meteorológica da Universidade do Estado do Mato Grosso, Campus II (agosto 2015 a agosto 2016).

Devido à alta variabilidade dos dados, houve a necessidade de transformação dos dados de contagem para cada espécie. As variáveis analisadas neste estudo foram submetidas inicialmente ao teste de Lilliefors para avaliar a normalidade dos dados. Como os dados não foram considerados normais utilizou-se a transformação $\log (x+1)$, com a finalidade de atingir os pressupostos dos testes paramétricos.

Realizou-se o teste de média para analisar a influência dos períodos climáticos (seco e chuva) sobre a abundância e riqueza. Foi utilizado o delineamento inteiramente casualisado em arranjo fatorial, sendo um DIC Fatorial 13 x 2 (13 espécies por dois períodos climáticos, com doze armadilhas/repetições). As médias foram submetidas à análise de variância e comparadas pelo teste $\mathrm{T}(\mathrm{p} \leq 0.05)$, utilizando-se o recurso computacional SISVAR, versão 4.0 (FERREIRA, 2000).

Com os dados quinzenais dos indivíduos coletados foi calculada a Correlação de Pearson (r) $(\mathrm{p} \leq 0.05)$, entre estas populações e a temperatura máxima, média, mínima, umidade relativa e precipitação 
pluviométrica. Este estudo foi realizado somente com as espécies que ocorreram na análise faunística como dominantes, muito abundantes, muito frequentes e constantes. Para esta análise foi utilizado o software R (R DEVELOPMENT CORE TEAM, 2012).

Realizaram-se a análise de variância e o teste de média pelo Teste $\mathrm{T}(\mathrm{p} \leq 0.05)$, para as médias dos dados transformados em log, dos diferentes fatores. O teste de média foi aplicado para verificar a diferença entre os dados, bem como agrupamentos de espécies quanto à sua distribuição dentre os períodos climáticos, em cada ambiente amostrado.

Foram calculados, ainda, os índices faunísticos de constância, dominância, frequência e abundância para os períodos de estiagem e, de chuva, por meio do software Anafau (MORAES et al., 2003). Foi calculada a Diversidade de Shannon-Wiener (H'), e Margalef $(\alpha)$, bem como a Equitabilidade (E), para os períodos climáticos de seca e de chuva no ambiente em questão.

A flutuação populacional foi analisada somente para as espécies identificadas que ocorreram na análise faunística como dominantes, muito abundantes, muito frequentes, e constantes. Calculou-se ainda, a dispersão espacial para as espécies de escolitíneos coletados no plantio de Eucalyptus urophylla $x$ Eucalyptus grandis, através dos indices de: Razão variância/média (I), Morisita (Iס) e Coeficiente de Green (Cx) (MORISITA, 1962; GREEN, 1966).

\section{RESULTADOS E DISCUSSÃO}

No período do levantamento foi coletado um total de 1.248 indivíduos, distribuídos em seis gêneros, com um total de 13 espécies. Os gêneros Criptocarenus, Hypotenemus e Xyleborus foram os que mais se destacaram no estudo, por apresentarem maior número de indivíduos coletados.

Segundo Dorval et al. (2007), o gênero Xyleborus apresenta o maior número de espécies com potencial de danos, mesmo que apresente espécies que possam ser consideradas benéficas, pois auxiliam na desrama natural de pequenos ramos, contribuindo na degradação de resíduos de madeira dentro de áreas reflorestadas.

Conforme a análise faunística na Tabela 1, 11 espécies foram dominantes e duas não dominantes. No parâmetro abundância, quatro espécies ocorreram como raras, cinco comuns e uma dispersa. Para frequência, cinco espécies ocorreram como pouco frequentes, cinco como frequentes e três como muito frequentes. No parâmetro constância, sete espécies apresentaram-se como constantes, três acessórias e três acidentais.

TABELA 1: Índices faunísticos para os escolitíneos coletados com armadilha etanólica de impacto em plantio de Eucalyptus urophylla $x$ Eucalyptus grandis, em Alta Floresta-MT (2015/2016).

TABLE 1: Faunal indices for bark beetle collected with ethanol trap impact on planting Eucalyptus urophylla $x$ Eucalyptus grandis in Alta Floresta, MT state, Brazil (2015/2016).

\begin{tabular}{|c|c|c|c|c|}
\hline Espécie & Dominâmica & Abundância & Frequência & Constância \\
\hline Cnesinus dryographus Sched $^{1}$ & ND & $\mathrm{r}$ & $\mathrm{PF}$ & $\mathrm{Z}$ \\
\hline $\begin{array}{l}\text { Criptocarenus diadematus } \\
\text { Eggers }^{1}\end{array}$ & $\mathrm{D}$ & $\mathrm{Ma}$ & MF & $\mathrm{W}$ \\
\hline $\begin{array}{l}\text { Criptocarenus heveae } \\
\text { Hagedorni }^{1}\end{array}$ & $\mathrm{D}$ & $\mathrm{Ma}$ & MF & W \\
\hline Criptocarenus seriatus Eggers ${ }^{1}$ & $\mathrm{D}$ & $\mathrm{Ma}$ & MF & $\mathrm{W}$ \\
\hline $\begin{array}{l}\text { Hypotenemus eruditus } \\
\text { Westwood }\end{array}$ & $\mathrm{D}$ & $\mathrm{c}$ & $\mathrm{F}$ & $\mathrm{W}$ \\
\hline $\begin{array}{l}\text { Hypotenemus bolivianus } \\
\text { Eggers }\end{array}$ & ND & $\mathrm{r}$ & $\mathrm{PF}$ & Z \\
\hline
\end{tabular}


TABELA 1: Continuação...

TABLE 1: Continued...

\begin{tabular}{lllll}
\hline Espécie & Dominâmica & Abundância & Frequência & Constância \\
\hline Hypotenemus seriatus Eichhoff & $\mathrm{D}$ & $\mathrm{c}$ & $\mathrm{F}$ & $\mathrm{Y}$ \\
Xyleborinus reconditus Schedl & $\mathrm{D}$ & $\mathrm{r}$ & $\mathrm{PF}$ & $\mathrm{Z}$ \\
$\begin{array}{l}\text { Xyleborus affinis Eichhoff } \\
\text { Xyleborus ferrugineus }\end{array}$ & $\mathrm{D}$ & $\mathrm{c}$ & $\mathrm{F}$ & $\mathrm{W}$ \\
$\begin{array}{l}\text { Fabricius } \\
\text { Xyleborus spinulosus }\end{array}$ & $\mathrm{D}$ & $\mathrm{c}$ & $\mathrm{F}$ & $\mathrm{Y}$ \\
$\begin{array}{l}\text { Blandford } \\
\text { Xylosandrus curtulus Eichhoff } \\
\text { Xylosandrus germanus }\end{array}$ & $\mathrm{D}$ & $\mathrm{c}$ & $\mathrm{F}$ & $\mathrm{W}$ \\
Blandford & $\mathrm{D}$ & $\mathrm{r}$ & $\mathrm{PF}$ & $\mathrm{Y}$ \\
\hline Equitabilidade $(\mathrm{E})$ & $\mathrm{d}$ & $\mathrm{d}$ & $\mathrm{PF}$ & $\mathrm{W}$ \\
0.80 & Margalef $(\alpha)$ & Shannon-Wiener $(\mathrm{H})$ & & \\
\hline
\end{tabular}

Em que: $\mathrm{D}=$ dominante; $\mathrm{ND}=$ não dominante; $\mathrm{A}=$ Abundância; $\mathrm{Ma}=$ muito abundante; $\mathrm{c}=$ comum; $\mathrm{d}=\mathrm{dispersa} ; \mathrm{r}=$ rara; $\mathrm{F}=$ Frequência; $\mathrm{MF}=$ muito frequente; $\mathrm{F}=$ frequente; $\mathrm{PF}=$ pouco frequente; $\mathrm{C}=\mathrm{Constância} ; \mathrm{W}=$ constante; $\mathrm{Y}$ = acessória; $\mathrm{Z}=$ acidental; ${ }^{1}$ indivíduos escolhidos para Correlação entre a flutuação populacional e fatores climáticos.

As espécies Criptocarenus diadematus, Criptocarenus heveae e Criptocarenus seriatus ocorreram como dominantes, muito abundantes, muito frequentes e constantes. Cnesinus dryographus e Hypotenemus bolivianus foram não dominantes, raras, pouco frequentes e acidentais. Em contrapartida, as espécies Hypotenemus eruditus, Xyleborus affinis e Xyleborus spinulosus ocorreram como dominantes, comuns, frequentes e constantes (Tabela 1).

A presença da espécie Criptocarenus heveae têm sido relatada por vários autores em diferentes fisionomias no Brasil. Dorval e Peres-Filho (2001) coletaram Criptocarenus heveae em vegetação do Cerrado no Mato Grosso. Silva et al. (2009) registraram essa espécie em uma área de floresta nativa em um corredor agroflorestal em Seropédica, Rio de Janeiro. Também no Rio de Janeiro, a espécie foi registrada por Silva (2012) em uma área de mangue. Já Machado (2013) relatou a presença da espécie em floresta nativa e povoamento de Pinus taeda em Santa Maria, Rio Grande do Sul. Esses registros demonstram assim a ampla distribuição da espécie em diferentes ambientes e regiões. Gusmão (2011) e Rocha (2010) em plantios de Eucalyptus spp. e área de Cerrado no município de Cuiabá-MT, observaram as espécies Hypotenemus eruditus e Criptocarenus diadematus como dominantes, muito abundantes, muito frequentes e constantes, diferindo deste trabalho.

Conforme a Tabela 1, a comunidade de escolitíneos avaliada apresentou uma equitabilidade (E) de espécies em 0,80, mostrando um índice de riqueza de Margalef $(\alpha)$ de 1.66, ficando com uma diversidade de Shannon-Wiener (H) em 2.07. Desta forma, os escolitineos do presente trabalho possuem uma melhor distribuição e uma maior diversidade dos indivíduos entre as espécies que compõem a comunidade se compararmos com Jorge (2014) em plantio do híbrido Urocam (Eucalyptus urophylla x Eucalyptus camaldulensis), no município de Chapada dos Guimarães-MT, que obteve uma Equitabilidade (E) anual de 0.54, Margalef $(\alpha)$ de 1.73 e Shannon-Wiener $(H)$ de 1.40 .

A flutuação populacional das principais espécies de Scolytinae coletadas em plantio de Eucalyptus urophylla x Eucalyptus grandis pode ser observada na Figura 2 

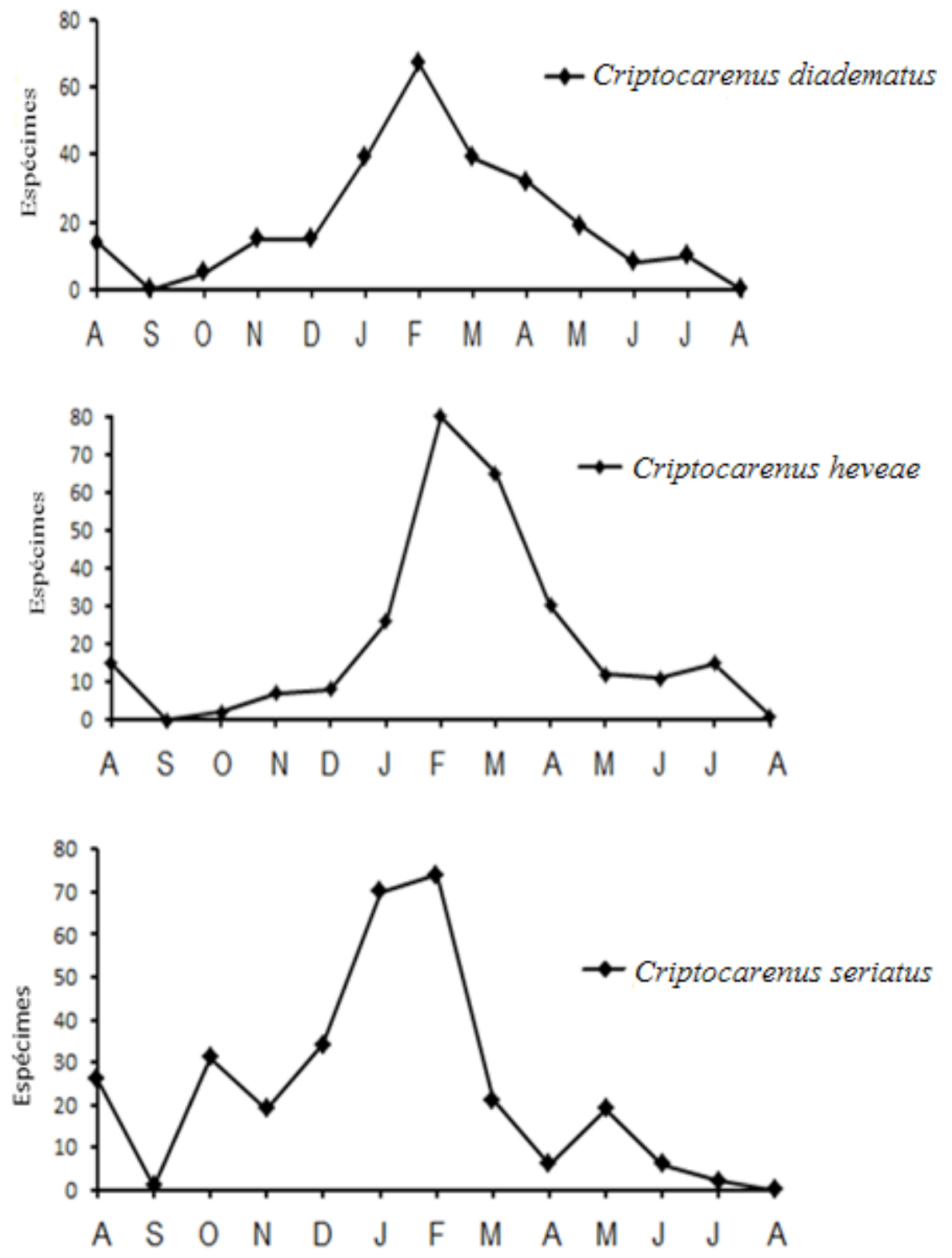

FIGURA 2: Flutuação populacional de três espécies de escolitíneos em plantio de Eucalyptus urophylla x Eucalyptus grandis no município de Alta Floresta-MT (2015/2016).

FIGURE 2: Population fluctuation of three bark beetle species in Eucalyptus urophylla $\mathrm{x}$ Eucalyptus grandis planting in the municipality of Alta Floresta, MT state, Brazil (2015/2016).

No plantio de Eucalyptus urophyllax Eucalyptus grandis, Criptocarenus diadematus e Criptocarenus heveae ocorreram com picos populacionais em fevereiro (período de chuva) e Criptocarenus seriatus apresentou uma grande elevação na quantidade de indivíduos coletados no mês de janeiro e fevereiro (período de chuva), março e outubro, caracterizado como período de estiagem na região, conforme Figura 2.

Esses resultados divergiram de alguns autores no estado de Mato Grosso. Jorge (2014), para a espécie Criptocarenus heveae, Dorval e Peres-Filho (2001), para a espécie Criptocarenus diadematus e Rocha et al. (2011b), para as espécies Criptocarenus seriatus e Criptocarenus diadematus, constataram picos populacionais e condições favoráveis para a sobrevivência das espécies mencionadas em períodos de baixa precipitação pluviométrica, diferindo do presente trabalho. No norte do estado do Mato Grosso chove mais que nas regiões em comparação, contudo, pode ser que os escolitineos iniciam seu voo no periodo das 
chuvas, explicando assim a diferença.

$\mathrm{Na}$ Tabela 2 apresenta-se a correlação entre flutuação populacional dos escolitíneos selecionados e fatores climáticos em plantio de Eucalyptus urophylla $x$ Eucalyptus grandis.

TABELA 2: Correlação entre a flutuação populacional dos escolitíneos selecionados e fatores climáticos, em plantio de Eucalyptus urophylla $x$ Eucalyptus grandis, Alta Floresta-MT (2015/2016).

TABLE 2: Correlation between the population fluctuation of selected bark beetle and climatic factors in Eucalyptus urophylla $x$ Eucalyptus grandis planting, Alta Floresta, MT state, Brazil (2015/2016).

\begin{tabular}{lllllll}
\hline Indiv./Ambientes & Média & Artcmax & Artcmin & Urmax & Urmin & Chuva \\
\hline Criptocarenus diadematus & $-0.59^{*}$ & $-0.67^{*}$ & $0.52^{\mathrm{NS}}$ & $0.50^{\mathrm{NS}}$ & $0.66^{*}$ & $0.63^{*}$ \\
Criptocarenus heveae & $-0.49^{\mathrm{NS}}$ & $-0.51^{\mathrm{NS}}$ & $0.38^{\mathrm{NS}}$ & $0.32^{\mathrm{NS}}$ & $0.50^{\mathrm{NS}}$ & $0.58^{*}$ \\
Criptocarenus seriatus & $-0.38^{\mathrm{NS}}$ & $-0.57^{*}$ & $0.43^{\mathrm{NS}}$ & $0.31^{\mathrm{NS}}$ & $0.51^{\mathrm{NS}}$ & $0.53^{\mathrm{NS}}$ \\
\hline
\end{tabular}

Em que: * significativo a 5\% de probabilidade pela correlação de Pearson; NS = não significativo.

No plantio de Eucalyptus urophylla $x$ Eucalyptus grandis, a população de Criptocarenus diadematus correlacionou-se negativamente com as temperaturas máxima e média, positivamente com a umidade relativa do ar mínima e com precipitação pluviométrica. A população de Criptocarenus heveae correlacionou-se positivamente com a precipitação pluviométrica. Houve correlação negativa com a população de Criptocarenus seriatus apenas com a temperatura máxima, conforme Tabela 2.

Os resultados apresentados neste estudo diferem de alguns estudos observados no estado de Mato Grosso. Trabalhos de Dorval e Peres Filho (2001) em Cerrado, no município de Cuiabá, mostraram correlação significativa negativa entre Criptocarenus heveae e temperatura mínima e precipitação pluviométrica; Criptocarenus diadematus para, umidade relativa do ar e precipitação pluviométrica; Criptocarenus seriatus para temperatura mínima, umidade relativa do ar e precipitação pluviométrica. Para a temperatura máxima, a correlação mostrou-se positiva. Rocha (2010) constatou em Cerrado, que a população de Criptocarenus diadematus correlacionou-se negativamente com a umidade relativa do ar. Murari (2012) verificou que o fator climático que apresentou maior influência na coleta das espécies de escolitíneos foi a umidade relativa do ar. Da mesma forma, Gusmão (2011) estudando quatro ambientes florestais distintos, no município de Cuiabá-MT, não encontrou significância com a precipitação pluviométrica. A diferença dos resultados observados neste estudo e dos autores citados pode estar relacionada ao fato das coletas terem sido realizadas em biomas diferentes, com caracteristicas ambientais também diversas, o que pode influenciar na distribuição das espécies durante $o$ ano.

Na Tabela 3 apresenta-se o teste de média entre as espécies de escolitíneos coletadas no plantio de Eucalyptus urophylla $x$ Eucalyptus grandis

TABELA 3: Teste de médias para os escolitíneos coletados no plantio de Eucalyptus urophylla $\mathrm{x}$ Eucalyptus grandis em Alta Floresta-MT (2015/2016).

TABLE 3: Testing of means for the bark beetle collected in Eucalyptus urophylla $x$ Eucalyptus grandis planting in Alta Floresta, MT state, Brazil (2015/2016).

\begin{tabular}{ccr}
\hline & \multicolumn{2}{c}{ Médias } \\
\cline { 2 - 3 } Espécies & Seca & Chuva \\
\hline Cnesinus dryographus & - & $0.08 \mathrm{cA}$ \\
Criptocarenus diadematus & $2.58 \mathrm{aB}$ & $17.08 \mathrm{aA}$ \\
\hline
\end{tabular}


TABELA 3: Continuação...

TABLE 3: Continued..

\begin{tabular}{ccc}
\hline & \multicolumn{2}{c}{ Médias } \\
\cline { 2 - 3 } Espécies & Seca & Chuva \\
\cline { 2 - 3 } Criptocarenus seriatus & $2.83 \mathrm{aB}$ & $21.75 \mathrm{aA}$ \\
Hypotenemus eruditus & $2.25 \mathrm{aB}$ & $9.08 \mathrm{bA}$ \\
Hypotenemus bolivianus & - & $0.33 \mathrm{cA}$ \\
Hypotenemus seriatus & - & $4.00 \mathrm{cA}$ \\
Xyleborinus reconditus & $0.08 \mathrm{aA}$ & $0.66 \mathrm{cA}$ \\
Xyleborus affinis & $2.75 \mathrm{aA}$ & $3.08 \mathrm{cA}$ \\
Xyleborus ferrugineus & $1.33 \mathrm{aB}$ & $7.66 \mathrm{bA}$ \\
Xyleborus spinulosus & $0.08 \mathrm{aA}$ & $1.83 \mathrm{cA}$ \\
Xylosandrus curtulus & $0.25 \mathrm{aA}$ & $1.50 \mathrm{cA}$ \\
Xylosandrus germanus & $1.33 \mathrm{aA}$ & $1.66 \mathrm{cA}$ \\
\hline
\end{tabular}

Médias seguidas da mesma letra maiúscula na linha e de mesma letra minúscula na coluna, não diferem entre si a 5\% de probabilidade pelo teste $\mathrm{T}$.

Nos talhões de Eucalyptus urophylla x Eucalyptus grandis não ocorreu diferença significativa entre espécies no período da seca. No período de chuva, obtiveram-se três agrupamentos de média sendo Criptocarenus seriatus, Criptocarenus heveae e Criptocarenus diadematus com as maiores médias, respectivamente. As espécies Hypotenemus eruditus e Xyleborus ferrugineus formaram um grupo de segunda importância quantitativa, sendo que as espécies Cnesinus dryographus, Hypotenemus bolivianus e Hypotenemus seriatus não obtiveram registro no período da seca. No período da chuva as espécies Cnesinus dryographus e Hypotenemus bolivianus foram as que apresentaram as médias mais baixas (Tabela 3).

A espécie Xyleborus ferrugineus também foi mais presente no período da chuva, corroborando com os estudos de Rocha et al. (2011a), em plantios de Eucalyptus camaldulensis Dehn. no município de Cuiabá, MT, sendo a espécie mais importante, pois apresentou maior quantidade de indivíduos e foi significativamente maior que as demais espécies nesse período.

Outras duas espécies: Criptocarenus seriatus e Hypotenemus eruditus formaram um grupo de segunda importância quantitativa, enquanto as demais espécies ocorreram com médias de coleta muito baixas, formando um terceiro agrupamento com diferentes espécies que não apresentaram diferenças estatísticas significativas entre si. Das médias analisadas entre os períodos climáticos foi possível notar que Criptocarenus diadematus, Criptocarenus heveae, Criptocarenus seriatus e Hypotenemus eruditus demonstraram diferenças significativas entre suas médias nos períodos de seca e de chuva (Tabela 3).

Cryptocarenus é um gênero tipicamente americano, com a maioria das espécies presentes na América do Sul. As fêmeas atacam árvores estressadas, broqueando ramos quebrados e danificados de pequeno porte, as espécies deste gênero são associadas a distúrbios ecológicos (WOOD, 1982).

Conforme Rodríguez (2016), em seu estudo realizado em diferentes sistemas vegetacionais, no município de Piracicaba-SP, o gênero Hypothenemus foi o mais abundante, representado por uma espécie, sendo semelhante com o presente trabalho. Para Dorval (2002), em plantios de Eucalyptus, a espécie Xyleborus affinis foi coletada em maior quantidade no período da chuva do que na seca, similarmente ao observado neste estudo.

Os dados apresentados na Tabela 4 demonstram os índices de dispersão para as espécies de escolitíneos coletados no plantio de Eucalyptus urophylla x Eucalyptus grandis. 
TABELA 4: Índices de dispersão dos escolitíneos coletados no plantio de Eucalyptus urophylla $x$ Eucalyptus grandis em Alta Floresta-MT (2015/2016).

TABLE 4: $\quad$ Scatter indices of the bark beetle collected at Eucalyptus urophylla $x$ Eucalyptus grandis planting in Alta Floresta, MT state, Brazil (2015/2016).

\begin{tabular}{cccc}
\hline Espécies & Morisita & Var-Media & Green \\
\hline Xylosandrus germanus & $1.72^{\text {agr }}$ & $3.44^{\mathrm{agr}}$ & $0.065^{\mathrm{agr}}$ \\
Hypotenemus eruditus & $1.25^{\mathrm{agr}}$ & $3.81^{\mathrm{agr}}$ & $0.023^{\mathrm{agr}}$ \\
Criptocarenus heveae & $1.75^{\mathrm{agr}}$ & $18.71^{\mathrm{agr}}$ & $0.068^{\mathrm{agr}}$ \\
Criptocarenus seriatus & $1.06^{\mathrm{agr}}$ & $2.70^{\mathrm{agr}}$ & $0.0061^{\mathrm{agr}}$ \\
Criptocarenus diadematus & $1.24^{\mathrm{agr}}$ & $6.69^{\mathrm{agr}}$ & $0.022^{\mathrm{agr}}$ \\
Xyleborus affinis & $1.21^{\mathrm{agr}}$ & $2.33^{\mathrm{agr}}$ & $0.019^{\mathrm{agr}}$ \\
Hypotenemus seriatus & $1.74^{\mathrm{agr}}$ & $4.85^{\mathrm{agr}}$ & $0.067^{\mathrm{agr}}$ \\
Xyleborus spinulosus & $1.56^{\mathrm{agr}}$ & $2.22^{\mathrm{agr}}$ & $0.05^{\mathrm{agr}}$ \\
Cnesinus dryographus & $1^{\mathrm{ale}}$ & $1^{\mathrm{ale}}$ & $0.018^{\mathrm{agr}}$ \\
Hypotenemus bolivianus & $1.2^{\mathrm{agr}}$ & $1.07^{\mathrm{agr}}$ & $0.084^{\mathrm{agr}}$ \\
Xyleborus ferrugineus & $1.92^{\mathrm{agr}}$ & $9.84^{\mathrm{agr}}$ & $0.028^{\mathrm{agr}}$ \\
Xylosandrus curtulus & $1.31^{\mathrm{agr}}$ & $1.57^{\mathrm{agr}}$ & $0.22^{\mathrm{agr}}$ \\
\hline Xyleborinus reconditus & $3.42^{\mathrm{agr}}$ & $2.32^{\mathrm{agr}}$ & \\
\hline
\end{tabular}

Em que: agr = agregado; ale = aleatório.

De acordo com a Tabela 4, o índice de Morisita (I $\delta$ ) alcançou valores acima de um, indicando uma distribuição agregada. Os valores da razão variância/média $(I)$ também foram superiores a um, demostrando agregação. Os valores do índice de Green $(\mathrm{Cx})$ também foram superiores a zero, sugerindo assim uma distribuição agregada (Tabela 4). Entretanto, a espécie Cnesinus dryographus apresentou uma distribuição espacial aleatória, provavelmente pelo baixo número de indivíduos coletados.

Esse padrão de agregação se explica porque muitas espécies de coleópteros, principalmente escolitíneos (besouros-da-casca), alimentam-se e agregam-se para acasalar na planta hospedeira, como um resultado da atração pelo feromônio sexual e a síntese do feromônio nos machos é estimulada pela alimentação na árvore hospedeira (WOOD, 1982; BORDEN, 2003).

\section{CONCLUSÕES}

Com base nos resultados obtidos, concluiu-se que a maioria das espécies de escolitíneos encontrou condições ambientais favoráveis no período de chuva, apresentando as maiores médias, bem como os maiores picos populacionais no período chuvoso.

Devido à presença de espécies-praga de Scolytinae importantes na área deste estudo, propõese buscar estratégias para desenvolvimento de Programas de Manejo Integrado de Pragas Florestais, contribuindo assim para a manutenção de um ambiente ecologicamente mais equilibrado e menos susceptível à ocorrência de surtos populacionais dessas coleobrocas. 


\section{AGRADECIMENTOS}

A Universidade Estadual de Mato Grosso (UNEMAT) e a CAPES pela bolsa de Mestrado do primeiro autor. Ao professor Dr. Eli Nunes Marques da Universidade Federal do Paraná pela identificação do material coletado.

\section{REFERÊNCIAS}

ALVARES, C. A. et al. Koopen's climate classification map for Brazil. Meteorologische Zeitschrift, Stuttgart, v. 22, n. 6, p. 711-728, 2014.

BACHA, C. J. C.; BARROS, A. L. M. Reflorestamento no Brasil: evolução recente e perspectivas para o futuro. Scientia Florestalis, Piracicaba, v. 66, p. 191-203, 2004.

BERTI FILHO, E. Cupins e Florestas. In: BERTI FILHO, E.; FONTES, L. R. Alguns aspectos atuais da biologia e controle de cupins. Piracicaba: FEALQ, 1995, p. 127-140.

BORDEN, J. H. Strategies and tactis for the use of semiochemicals against forest insect pests in North America. In: LUMSDEN, R. D.; VAUGHN, J. L. (Ed.). Pest management: biologically based technologies. Washington: American Chemistry Society, 2003. p. 265-279.

CARVAlHO, A. G. Armadilha modelo Carvalho-47. Floresta e Ambiente, Seropédica, v. 5, n. 1, p. 225-227, 1998.

DORVAL, A. Levantamento populacional de coleópteros com armadilhas etanólicas em plantios de eucaliptos e em uma área com vegetação de cerrado no município de Cuiabá, Estado de Mato Grosso. 2002. 143 f. Tese (Doutorado em Engenharia Florestal) - Universidade Federal do Paraná, Curitiba, 2002. DORVAL, A. et al. Infestação de coleobrocas em madeiras de Eucalyptus spp. em Cuiabá, Estado de Mato Grosso. Revista de Agricultura, Piracicaba, v. 82, n. 2, p. 134-141, 2007.

DORVAL, A.; PERES FILHO, O. Levantamento e flutuação populacional de coleópteros em vegetação do cerrado da Baixada Cuiabana, MT. Ciência Florestal, Santa Maria, v. 11, n. 2, p. 170-182, 2001.

DORVAL, A.; PERES FILHO, O.; MARQUES, E. N. Levantamento de Scolytidae (Coleoptera) em plantações de Eucalyptus spp. em Cuiabá, Estado de Mato Grosso. Ciência Florestal, Santa Maria, v. 14, n. 1, p. 47-58, 2004.

FERREIRA, D. F. Análise estatística por meio do SISVAR (Sistema para Análise de Variância) para Windows versão 4.0. In: REUNIÃO ANUAL DA REGIÃO BRASILEIRA DA SOCIEDADE INTERNACIONAL DE BIOMETRIA, 45., 2000, São Carlos. Anais... São Carlos: UFSCar, 2000. p. 255-258.

FONSECA, S. M. et al. Manual Prático de melhoramento genético do eucalipto. Viçosa, MG: UFV, 2010. 200 p.

GARLET, J. et al. Danos provocados por coró-das-pastagens em plantas de eucalipto. Ciência Rural, Santa Maria, v. 39, p. 575-576, 2009.

GONÇALVES, F. G. et al. Coleópteros broqueadores de madeira em ambiente natural de Mata Atlântica e em plantio de eucalipto. Pesquisa Florestal Brasileira, Colombo, v. 34, n. 79, p. 245-250, 2014.

GREEN, R. H. Measurement of non-randomness in spatial distributions. Researches on Population Ecology, Kyoto, v. 8, n. 1, p. 1-7, 1966.

GUSMÃO, R. S. Analise faunística de Scolytidae (Coleoptera) coletadas com armadilhas etanólicas com e sem porta isca em Eucalyptus ssp. em área de cerrado no município de Cuiabá - MT. 2011. 47 f. Dissertação (Mestrado em Ciências Florestais e Ambientais) - Universidade Federal de Mato Grosso, Cuiabá, 2011.

INDÚSTRIA BRASILEIRA DE ÁRVORES. Anuário estatístico 2016 ano base 2015. São Paulo: IBÁ, 2016.

JORGE, V. C. Influência de diferentes concentrações de etanol para a coleta de Scolytinae. 2014. 70 f. Dissertação (Mestrado em Ciências Florestais e Ambientais) - Universidade Federal de Mato Grosso, Cuiabá, 2014.

LIMA, A. M. C. Insetos do Brasil. Rio de Janeiro: Escola Nacional de Agronomia, 1956. v. 10. 373 p. MACHADO, L. M. Determinação da altura de voo de escolitídeos em mata nativa e em povoamento de Pinus taeda. 2013. 57 f. Dissertação (Mestrado em Engenharia Florestal) - Universidade Federal de 
Santa Maria, Santa Maria, 2013.

MALUCHE, C. R. D. et al. Fauna edáfica como bioindicadora da qualidade do solo em pomares de macieiras conduzidos nos sistemas orgânico e convencional. In: CONGRESSO BRASILEIRO DE AGROECOLOGIA, 1., 2003, Porto Alegre. Anais... Porto Alegre: EMATER/RS; ASCAR, 2003. CD-ROM.

MEURER, E. et al. Scolytinae (Coleoptera, Curculionidae) associados a diferentes fitofisionomias no Pantanal de Cáceres, Mato Grosso. Acta Biológica Paranaense, Curitiba, v. 42, n. 1-4, p. 195-210, 2013. MORAES, R. C. B. et al. Software para análise estatística - ANAFAU. In: SIMPÓSIO DE CONTROLE BIOLÓGICO, 8., 2003, São Pedro. Resumos... Piracicaba: ESALQ; USP, 2003. p. 195.

MORISITA, M. I $\delta$ - index, a measure of dispersion of individuals. Researches on Population Ecology, Kyoto, v. 4, n. 1, p. 1-7, 1962.

MURARI, A. B. et al. Modelo de armadilha etanólica de interceptação de vôo para captura de escolitídeos (Curculionidae: Scolytinae). Pesquisa Florestal Brasileira, Colombo, v. 32, n. 69, 2012.

NAKANO, O.; LEITE, C. A. Armadilhas para insetos: pragas agrícolas e domésticas. Piracicaba: Fundação de Estudos Agrários Luiz de Queiroz, 2000. 76 p.

R DEVELOPMENT CORE TEAM. R: a language and environment for statistical computing. Vienna: R Foundation for Statistical Computing, 2012.

ROCHA, J. R. M. et al. Análise da Ocorrência de Coleópteros em Plantios de Eucalyptus camaldulensis Dehn. em Cuiabá, MT. Floresta e Ambiente, Seropédica, v. 18 n. 4 p. 343-352, 2011 a.

ROCHA, J. R. M. et al. Coleópteros (Bostrichidae, Platypodidae e Scolytidae) em um fragmento de cerrado da baixada Cuiabana. Ambiência, Guarapuava, v. 7 n. 1 p. 89-101, 2011 b.

ROCHA, J. R. M. Ocorrência e dinâmica populacional de Scolytidae, Bostrichidae e Platypodidae em povoamentos de eucaliptos e fragmentos de cerrado, no município de Cuiabá - MT. 2010. $63 \mathrm{f}$. Dissertação (Mestrado) - Universidade Federal de Mato Grosso, Cuiabá, 2010.

RODRÍGUEZ, C. A. S. Estrutura da vegetação e sua relação com a diversidade, abundância e similaridade de coleópteros bioindicadores em diferentes sistemas vegetacionais, Piracicaba, SP. 2016. 125 f. Dissertação (Mestrado em Ciências) - Escola Superior de Agricultura "Luiz de Queiroz", Universidade de São Paulo, Piracicaba, 2016.

SILVA, C. O. Ocorrência de scolytinae no ambiente e na madeira de cinco espécies florestais em manguezal. 2012. 43 f. Dissertação (Mestrado em Ciências) - Instituto de Florestas, Universidade Federal Rural do Rio de Janeiro, Seropédica, 2012.

SILVA, D. G. et al. Entomofauna associada a remanescentes de mata atlântica conectados por um corredor ecológico agroflorestal. In: CONGRESSO BRASILEIRO DE SISTEMAS AGROFLORESTAIS,7., 2009, Luziânia. Anais... [s. 1.: s. n.], 2009. p. 2-4.

SILVA, M. M. Diversidade de insetos em diferentes ambientes florestais no município de Cotriguaçu, estado de Mato Grosso. 2009. 111 f. Dissertação (Mestrado em Ciências Florestais) - Universidade Federal de Mato Grosso, Cuiabá, 2009.

WOOD, S. L. The bark and ambrosia beetles of North and Central America (Coleoptera: Scolytidae), a taxonomic monograph. Cambridge: Harvard University, 1982. 1359 p. (Great Basin Naturalist Memoirs, n. 6). 